\title{
Creating a graft-friendly environment for stem cells in diseased brains
}

\author{
Robert Y.L. Tsai \\ Institute of Biosciences and Technology, Houston, Texas, USA and Department of Molecular and Cellular Medicine, Texas A\&M University Health Science Center, College Station, Texas, USA.
}

\begin{abstract}
Most of the adult CNS lacks regenerative activity in terms of both neuron birth and neurite outgrowth. While this regeneration-unfriendly environment of the adult CNS may preserve the existing neuronal circuitry that takes years to develop in higher organisms, it also poses a major obstacle for CNS repair later in life. In this issue of the JCI, Song et al. report on their development of a strategy that uses region-specific and molecularly engineered astrocytes to turn an unfavorable brain environment into a favorable one for engrafted neural stem/progenitor cells (NSC/NPCs). In a rat model of Parkinson's disease (PD), cografting NPCs with midbrainderived astrocytes engineered to overexpress the transcription factors Nurr1 and Foxa2 promotes maturation and survival of the graft, resulting in therapeutic improvement. The results of this study raise the prospect of using modified astrocytes to improve the survival, maturation, and integration of engrafted NSC/NPCs as a restorative treatment for PD.
\end{abstract}

\section{Organ preservation versus} regeneration: the good, the bad, and the ugly

Most of the adult CNS lacks the ability to regenerate in a physiologically meaningful manner, even though adult neurogenesis can occur in a few select sites, such as the subventricular zone and the dentate gyrus. As the electrical signal from a source neuron is transmitted by its axon, which sometimes must project several feet to reach the downstream target, the lack of neural regeneration in the adult brain and spinal cord has both advantages and disadvantages.

The good. For the human CNS, it takes years to establish neuronal pathways (or tracts) in their final shapes and forms. To continually produce the guiding cues that are only needed during development would be uneconomical on the one hand and might invite the risk of making erroneous connections on the other hand. In this regard, limited renewal is beneficial for the preservation of the anatomical integrity of already-established neuronal circuitry and the prevention of aberrant wiring.

The bad. Due to the changes that occur in the CNS environment beginning with the embryo and continuing to adulthood, reestablishing accurate, long-range projection of neuronal processes from scratch in the adult CNS becomes inconceivably challenging, if not impossible. Unfortunately, the paucity of regeneration in most parts of the adult brain makes cell replacement the only restorative treatment for diseases defined by irreversible neuronal loss, such as traumatic brain and spinal cord injury, Parkinson's disease (PD), Alzheimer's disease (AD), amyotrophic lateral sclerosis (ALS), and many more. While the idea of using stem cells as a universal source for restorative cell therapies is attractive, clinical application of such cell-based approaches in patients is not so straightforward. There are several major issues, including pervasiveness of the lesion, inconsistency of transplanted cells

Related Article: p. 463

Conflict of interest: The author has declared that no conflict of interest exists.

Reference information: / Clin Invest. 2018;128(1):116-119. https://doi.org/10.1172/JCI98490.

faithfully differentiating into the desired cell types and reconnecting with their rightful targets, and limited survival of the donor cells and their therapeutic effects, that hamper successful application of stem cell therapies in the clinic. In this capacity, the difficulty of applying cell-based therapies to neurological disease treatment is reflected in the failed outcomes of several preclinical trials that used human neural stem/progenitor cells (NSC/NPCs) to treat either spinal cord injury (1) or AD (2).

The ugly. Compared with spinal cord injury or $\mathrm{AD}, \mathrm{PD}$ is considered to be relatively amenable to cell-replacement therapies because its primary pathology - the loss of dopaminergic (DA) neurons - is confined to the substantia nigra and the length of the involved neural circuitry (the nigrostriatal and striatonigral pathways) is relatively short. The distance between grafted DA neurons and their targets can be further reduced by direct transplantation of donor cells into the striatum. The therapeutic benefit of intrastriatal transplantation of fetal mesencephalic tissues in PD rats has been established since 1979 $(3,4)$, and clinical trials to evaluate fetal tissue transplantation in PD patients began in 1987. Three decades later, cell therapies for PD treatment have yet to be approved by the FDA, largely owing to several ethical and practical issues (5). A great deal of effort has been focused on deriving donor cells from human embryonic stem cells (6) or from induced pluripotent stem cells (iPSCs) to resolve ethical issues surrounding the use of fetal tissues (7). So far, it has largely been ignored that the host brain often becomes inhospitable to grafted cells as a result of procedure-induced immunological responses that polarize the endogenous host glia from an antiinflammatory, neurotrophic form to a proinflammatory, cytotoxic form. Unfortunately, this hostile environment for engrafted NSC/NPCs can persist long after the transplantation and detrimentally affect the survival and function of these cells (8). 


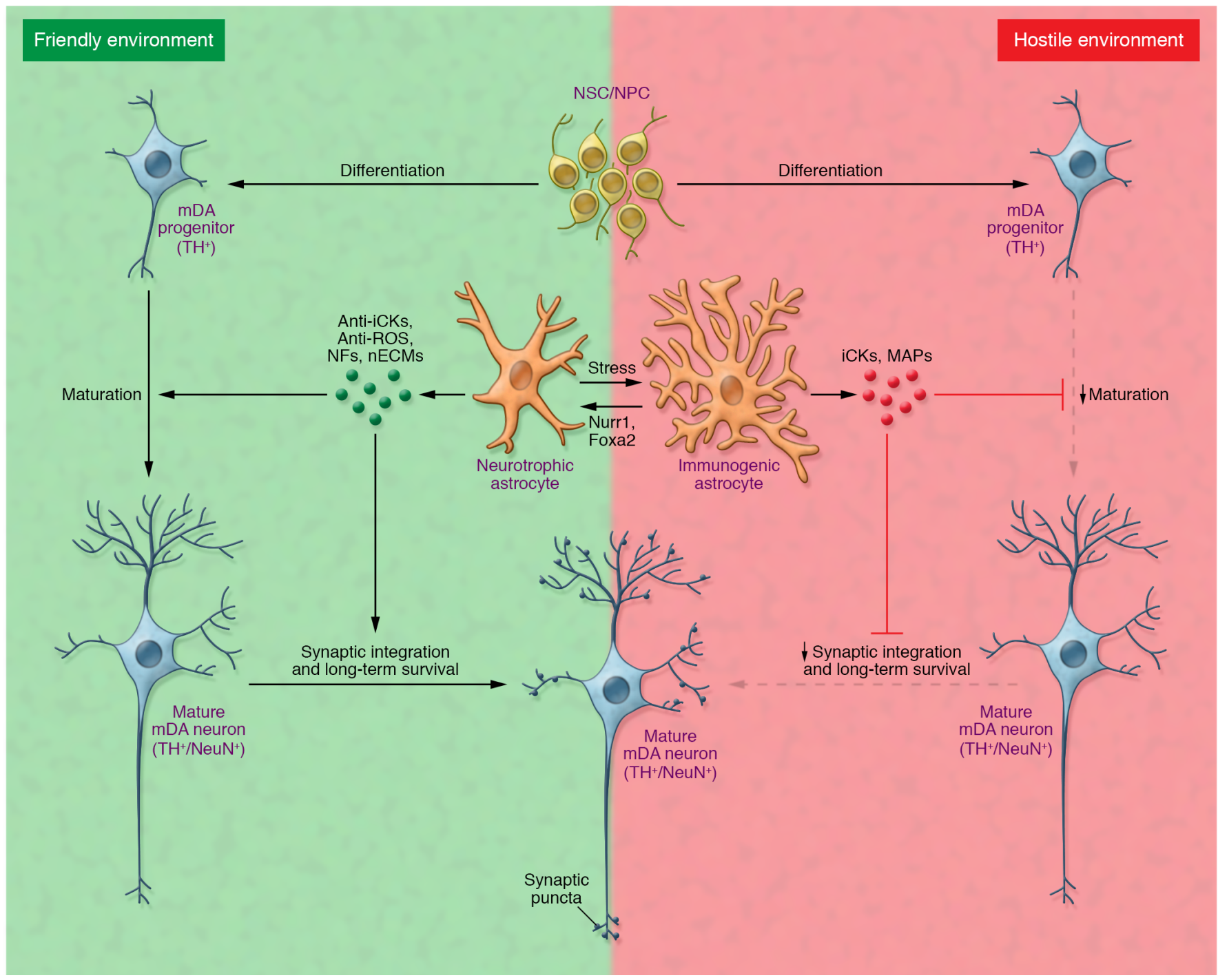

Figure 1. Host environment influences outcome of NSC/NPC transplantation in a rat model of PD. NSC/NPCs transplanted along with neurotrophic astrocytes create a friendly environment that allows their differentiation and maturation into mDA neurons. Neurotrophic astrocytes release several classes of molecules that promote mDA neuron differentiation and support long-term survival of these neurons. These include antiinflammatory cytokines (anti-iCKs), such as INF- $\alpha$, INF- $\beta$, IL-1R, CCL17, and CCL22; anti-ROS molecules, including SOD3 and GPX; neurotrophic factors, such as GDNF, SHH, BDNF, NTF3, and Wnts; and neurotrophic ECM proteins, including FN1, COL6A2, ITG $\beta 4$, ITG $\alpha \mathrm{M}$, THBS1, and GPC. The presence of stress introduced by the transplantation procedure can cause neurotrophic astrocytes to become immunogenic, creating a hostile environment for transplanted NSC/ NPCs. Immunogenic astrocytes hinder the maturation and survival of mDA progenitors through the production of proinflammatory cytokines (iCKs), such as IL-1 1 , TNF- $\alpha$, iNOS, IL-6, and CXCL11, and myelin-associated proteins (MAP), including MBP, MOG, and MAG. Induced expression of Nurr1 and Foxa2 tips astrocytes toward a neutrophic phenotype, thereby promoting the maturation and survival of donor cell-derived mDA neurons. TH, tyrosine hydroxylase; nECM, neurotrophic ECM.

\section{Cografting stem cells with astrocytes: the good neighbor policy}

In this issue, Song et al. report on their use of the 6-hydroxydopamine-induced lesion rat model of $\mathrm{PD}$ to address the idea that astrocytic neighbors may play a key role in determining the therapeutic outcome of engrafted NPCs (9). Animals cografted with NPCs prepared from rodent embryonic ventral midbrain (VM) and carefully selected astrocytes, such as those isolated from postnatal VM, exhibited marked and persistent improvements in the survival of engrafted NPCs, the differentiation and maturation of donor NPCs into midbrain DA (mDA) neurons, and the synaptic integration of donor-derived mDA neurons with the host striatal neurons. The overarching take-home message of this study, that the phenotype of neighboring astrocytes determines the outcome of transplanted NPCs, is based on two key findings. First,
Song et al. systematically determined the type of astrocytes that yield the best therapeutic outcome of stem cell therapy. It turns out that the regional identity of astrocytes matters. The authors first showed that cografting VM-derived astrocytes was better than cografting cortex-derived (Ctx-derived) astrocytes for improving the therapeutic outcome of VM-derived NPC transplantation in PD rats. Since VM astrocytes, like mDA neurons, are known to express high lev- 
els of the transcription factors Nurr1 and Foxa2, Song and colleagues engineered VM astrocytes to overexpress Nurr1 and Foxa2 and found that cografting of these genetically modified VM astrocytes improved the differentiation, maturation, synaptic integration, and survival of grafted NPCs in vivo. Arguably, the most exciting feature of this astrocyte cografting approach is the duration of the behavioral improvement and the maintenance of the midbrain identity of grafted cells, both of which were shown to last up to six months after transplantation.

\section{Turning immunogenic astrocytes into neurotrophic astrocytes: Dr. Jekyll and Mr. Hyde}

The next question is, why are all astrocytes not equal in exerting benefit to cografted NPCs? This question is addressed by the second key finding of this study - the elucidation of the mechanism that underlies the beneficial effect of cografting astrocytes. Song and colleagues compared the gene-expression profiles between astrocytes and Ctx-NPCs, VM-astrocytes and Ctx-astrocytes, and Nurr1+Foxa2-engineered VM-astrocytes and nonengineered VM-astrocytes. Regeneration-friendly astrocytes consistently exhibited lower expression of several proinflammatory cytokines and myelin-associated proteins and higher expression of neurotrophic factors, antiinflammatory cytokines, ROS scavengers, and neurotrophic extracellular matrix proteins (Figure 1). Conversely, proinflammatory, immunogenic astrocytes, which contribute to the hostile brain environment, secreted higher levels of proinflammatory cytokines and myelin-associated proteins. These findings indicate that only the neurotrophic, antiinflammatory form of astrocytes is conducive to the survival, maturation, and functional integration of transplanted VM-NPCs, whereas proinflammatory, immunogenic astrocytes are detrimental to the cause.

\section{Beyond PD: mission impossible}

The leading pathology of PD is the loss of DA neurons in the substantia nigra. Hence, the treatment objective for PD is simply to restore DA-secreting neurons or their functional equivalents at the target site - the striatum. Because mDA neurons belong to the extrapyramidal system, which functions to mod- ulate rather than to dictate the motor function, the requirement for regulated firing and exact wiring is not as stringent as that required for pyramidal neurons in the motor cortex. For these reasons, PD has always been an ideal candidate for cell-based therapies. Nevertheless, even for PD, the integration of donor-derived $\mathrm{mDA}$ neurons with host striatal neurons still plays an important role in the long-term survival of donor-derived mDA neurons and continuation of symptomatic recovery. For brain and spinal cord injury, the long-range connectivity between upper motor neurons in the motor cortex and lower motor neurons in the anterior horn of the spinal cord is undoubtedly the most critical determinant for functional restoration. As it may be impossible for reborn axons to navigate and rebuild the same path that they once had, a more realistic objective for treating CNS injury may be a reduction in the retrograde loss of neurons as well as facilitation of neurite regrowth across the lesion site before loss of the existing tract. Another challenge for most neurodegenerative diseases is the extent of pathological involvement, which may become too diffuse for cell transplantation to be feasible. In this case, diagnosis of disease at the earliest possible stage and stopping its progression will be the outcome-deciding factor. Despite all the challenges and complexity, the fundamental concept put forth in this study by Song et al. may still be broadly applicable, as grafting the right type of astrocytes by themselves may attenuate the underlying pathology via multiple pathways, such as eliminating pathological protein aggregates $(10,11)$.

\section{Future for cell-based therapy: tomorrow never dies}

The road to finding the much-coveted restorative cures for neurological diseases defined by irreversible neuronal loss is paved with many obstacles, and there are many questions that need to be answered. From a functional and mechanistic standpoint, all candidate genes (Figure 1) that have been identified based on their differential expression in various paradigms and/or their previously published roles in mDA neuron development will need to be studied to determine their actual contri- butions to either the neurotrophic, antiinflammatory environment or the proinflammatory environment. Such evaluation will require each gene to be individually analyzed, similar to what has been done for nucleostemin in stem cell self-renewal (12). From a therapeutic standpoint, the immediate work ahead is to determine the long-term survival of the grafted NPCs beyond the six-month period evaluated by Song and colleagues. This determination should preferably be done using human cells transplanted into humanized animal models. In addition, the native brain environment is made up not only of astrocytes but also of a community of various glial cell types, including microglia. It is possible that cografting a multiplex of glial cells may improve the therapeutic outcome of transplanted NPCs more than what was observed in this study by Song et al. Other areas of interest include understanding how to prevent the spontaneous reversion of neurotrophic astrocytes to proinflammatory astrocytes over time, how to avoid the overdose of cell therapy, and how to deal with the widespread lesion associated with most neurodegenerative disorders. Another concern with putting normal cells into a diseased environment is the possibility that the underlying pathology, such as the $\alpha$-synuclein aggregates in PD, may be transmitted to the donor cells after an extended period of time $(13,14)$. Finally, strategies to help donor-derived neurons to reestablish the correct long-range connections in the adult brain will have to be addressed for replacing neurons whose primary functions rely on such connectivity. Similarly, the integration of donor cells with their upstream neurons will no doubt play an important role in securing appropriate physiological regulation of their activity and neurotransmitter release. Despite these looming obstacles, Song and colleagues have taken a significant step forward by showing that one key to success may reside in having good neighbors, a concept that seems fundamentally simple and yet has not been explored quite enough. Although the road ahead remains long, one can at least take comfort in knowing that when venturing into a hostile territory, choosing the right company can go a long way and that many missions, however impossible they may seem at the beginning, turn out to be accomplishable in the end. 


\section{Acknowledgments}

The author would like to thank Laura Wu (University of Texas Medical Branch, Department of Neurology, Galveston, Texas, USA) for helpful discussion on this Commentary and to acknowledge the funding support to his laboratory by the NIH (AG052006, CA201988) and the Cancer Prevention Research Institute of Texas (RP170179).

Address correspondence to: Robert Y.L. Tsai, Institute of Biosciences and Technology, Texas A\&M University Health Science Center, 2121 W. Holcombe Blvd., Houston, Texas 77030, USA. Phone: 713.677.7690; Email:rtsai@ibt.tamhsc.edu.

1. Anderson AJ, Piltti KM, Hooshmand MJ, Nishi RA, Cummings BJ. Preclinical efficacy failure of human CNS-derived stem cells for use in the pathway study of cervical spinal cord injury. Stem Cell Reports. 2017;8(2):249-263.

2. Marsh SE, et al. HuCNS-SC human NSCs fail to differentiate, form ectopic clusters, and provide no cognitive benefits in a transgenic model of Alzheimer's disease. Stem Cell Reports. 2017;8(2):235-248.

3. Perlow MJ, Freed WJ, Hoffer BJ, Seiger A, Olson L, Wyatt RJ. Brain grafts reduce motor abnormalities produced by destruction of nigrostriatal dopamine system. Science. 1979;204(4393):643-647.

4. Björklund A, Stenevi U. Reconstruction of the nigrostriatal dopamine pathway by intracerebral nigral transplants. Brain Res. 1979;177(3):555-560.

5. Lindvall O. Clinical translation of stem cell transplantation in Parkinson's disease. J Intern Med. 2016;279(1):30-40.

6. Cyranoski D. Trials of embryonic stem cells to launch in China. Nature. 2017;546(7656):15-16.

7. Kikuchi T, et al. Human iPS cell-derived dopaminergic neurons function in a primate Parkinson's disease model. Nature. 2017;548(7669):592-596.

8. Barker RA, Widner H. Immune problems in central nervous system cell therapy. NeuroRx. 2004;1(4):472-481.

9. Song JJ, et al. Cografting astrocytes improves cell therapeutic outcomes in a Parkinson's disease model. J Clin Invest. 2018;128(1):463-482.

10. Lindström V, et al. Extensive uptake of $\alpha$-synuclein oligomers in astrocytes results in sustained intracellular deposits and mitochondrial damage. Mol Cell Neurosci. 2017;82:143-156.

11. Loria F, et al. $\alpha$-Synuclein transfer between neurons and astrocytes indicates that astrocytes play a role in degradation rather than in spreading. Acta Neuropathol. 2017;134(5):789-808.

12. Tsai RY. Turning a new page on nucleostemin and self-renewal. J Cell Sci. 2014;127(pt 18):3885-3891.

13. Kordower JH, Chu Y, Hauser RA, Freeman TB, Olanow CW. Lewy body-like pathology in long-term embryonic nigral transplants in Parkinson's disease. Nat Med. 2008;14(5):504-506.

14. Li JY, et al. Lewy bodies in grafted neurons in subjects with Parkinson's disease suggest host-to-graft disease propagation. Nat Med. 2008;14(5):501-503. 